\title{
Randomized Controlled Trial between Levetiracetam and Phenobarbital in the Treatment of Neonatal Seizure due to Perinatal Asphyxia
}

ABU FAISAL MD PERVEZ ${ }^{1}$, MD. FAKHRUL AMIN BADAL ${ }^{2}$, S.M. NURUN NABI ${ }^{3}$, MOHAMMAD KAMRUL HASSAN SHABUJ ${ }^{4}$, SANJOY KUMER DEY ${ }^{5}$, M.A MANNAN ${ }^{6}$, MOHAMMOD SHAHIDULLAH ${ }^{7}$

\begin{abstract}
:
Background: Seizure occurs more frequently in neonatal period and incidence of seizure is $50 \%-68 \%$ in perinatal asphyxia. At present phenobarbital is the drug of choice for treating neonatal seizure, which has some adverse effects on neurodevelopment status. Levetiracetam is a novel antiepileptic agent well-tolerated and effective in focal, generalized and neonatal seizure as well and lacks the adverse effects like phenobarbital. The present study was undertaken to compare the safety and efficacy of levetiracetam to phenobarbital in the treatment of neonatal seizure due to perinatal asphyxia.

Methodology: This interventional study (Randomized Controlled Trial) was conducted in Department of Neonatology, Bangabandhu Sheikh Mujib Medical University (BSMMU), Dhaka and Dhaka Medical College Hospital, Dhaka, Bangladesh from 1st January' 2014 to 30th June' 2015. Intravenous levetiracetam injection, $50 \mathrm{mg} / \mathrm{kg}$ loading followed by $10 \mathrm{mg} / \mathrm{kg} 8$ hourly maintenance was used and phenobarbital intravenous 20-40 mg/kg loading and $2.5 \mathrm{mg} / \mathrm{kg} / \mathrm{dose} 12$ hourly maintenance was given as per institutional protocol.

Results: Sixty-nine term asphyxiated neonates (intention to treat population) provided analyzable data. Seizure control was found significantly higher $(p=0.011)$ higher in levetiracetam group in comparison to phenobarbital group (71\% vs $40 \%)$. Need for more than one drug was significantly lower in levetiracetan group $(p=0.011)$. Adverse effects were found significantly ( $p=0.001)$ lower in levetiracetam group ( $9 \%$ vs $43 \%)$. No serious adverse effect was observed in any group and most common adverse effect was somnolence in both group followed by irritability. Restlessness, sedation and shallow breathing were found only in phenobarbital group.

Conclusion: Levetiracetam is more effective and safe in comparison to phenobarbital in the treatment of neonatal seizure due to perinatal asphyxia.
\end{abstract}

Key words: Seizure, Perinatal Asphyxia, Levetiracetam, Phenobarbitone.

1. Assistant Professor of Neonatology, Department of Paediatrics, Faridpur Medical College, Faridpur, OSD, DGHS, Dhaka.

2. RP (Paediatrics), Sheikh Hasina Medical College Hospital, Tangail.

3. Registrar, Department of Paediatrics, Rangpur Medical College, Rangpur.

4. Assistant Professor, Department of Neonatology, Bangabandhu Sheikh Mujib Medical University (BSMMU), Dhaka.

5. Associate Professor, Department of Neonatology, BSMMU, Dhaka.

6. Professor, Department of Neonatology, BSMMU, Dhaka.

7. Professor and Chairman, Department of Neonatology, BSMMU, Dhaka.

Correspondence: Dr. Abu Faisal Md. Pervez, Assistant professor of Neonatology, Department of Paediatrics, Faridpur Medical College, Faridpur. OSD, DGHS, Dhaka. Mobile: +8801712025076 Received: 10 February 2018 Accepted: 21 June 2018

\section{Introduction:}

Seizure occurs more frequently in neonatal period than at any other time of life and incidence of neonatal seizure ranges from 0.95 to 3.5 per thousand live birth. ${ }^{1}$ According to Brown et al and Finer et al, incidence of seizure is $50 \%-68 \%$ in perinatal asphyxia. ${ }^{2}$ At present phenobarbital is the drug of choice for treating neonatal seizure, which has some adverse effects on neurodevelopment. ${ }^{3}$ In addition, the known risk of cognitive impairment of phenobarbital in infants and toddlers should be considered. ${ }^{3}$ Levetiracetam is a novel antiepileptic agent well-tolerated and effective in focal, generalized and neonatal seizure as well and currently licensed as adjunctive therapy in the treatment of children and 
infants with epilepsy starting from 1 month of age in partial onset seizures, and already licensed in children aged 4 years or above for other indications. Studies showed its efficacy $71 \%-86 \%{ }^{4,5}$ whereas efficacy was found only $29 \%-85 \%$ in case of Phenobarbital. ${ }^{6,7}$ More over levetiracetam is rapidly and completely absorbed after oral administration. ${ }^{8}$ In a study by Maitre et al Levetiracetam showed better neurodevelopmental outcome in comparison to phenobarbitone ${ }^{9}$ levetiracetam has not been found to increase neuronal apoptosis in animal models. ${ }^{10}$ Prospective studies with small patient groups in infants and very young children revealed similar results. ${ }^{11,12}$ Levetiracetam is increasingly being used as an antiepileptic drug in the neonatal period. Use of levetiracetam in term and preterm neonates with rarely observed adverse effects were found in an analysis of surveys from neonatologists and pediatric neurologists. ${ }^{13}$ Levetiracetam may be an alternative for hypoxia induced neonatal seizure. No such sutdy was done comparing safety and efficacy of levetiracetam with phenobarbitone in seizure due to perinatal asphyxia. So this study is intended to evaluate the efficacy and side effects of levetiracetam which may open a new frontier of neonatal seizure management and may help to tailor new guideline.

\section{Materials and Methods}

It was a randomized controlled trial conducted in the Department of Neonatology, Bangabandhu Sheikh Mujib Medical University (BSMMU), Dhaka and Dhaka Medical College Hospital, Dhaka, Bangladesh from 1st January, 2014 to 30st June, 2015 with prior institutional review board (IRB) approval. Term neonates, aged up to 48 hours, admitted in Neonatal Intensive Care Unit with history of perinatal asphyxia and clinical seizure (both before and after admission) diagnosed by doctors and those have given written consent were included in the study. Convulsion management was given according to institutional protocol (Maintenance of temperature, airway, breathing, circulation, correction of hypoglycemia \& hypocalcaemia) except the anticonvulsants. For seizure control in neonates with active convulsion either levetiracetam or phenobarbital was allocated according to the randomization. Levetiracetam receivers were in intervention group and phenobarbital receivers were in control group. Levetiracetam was administered intravenous as a loading dose of $50 \mathrm{mg} / \mathrm{kg}$. Maintenance was administered intravenous at a dose of $10 \mathrm{mg} / \mathrm{kg} 8$ hourly. All bolus and maintenance doses were diluted with normal saline to a concentration of $20 \mathrm{mg} / \mathrm{ml}$ and boluses were administered over 15 minutes. Phenobarbital loading was given at a dose of 20-40 $\mathrm{mg} / \mathrm{kg}$ intravenously and maintenance doses were given $2.5 \mathrm{mg} / \mathrm{kg} /$ dose 12 hourly according to institutional protocol. When convulsion stopped completely and not recurred within 48 hours then it was considered as convulsion control and maintenance was followed up upto 48 hours. Adverse effects like life threatening conditions as well as somnolence, irritability, insomnia, hypersomnia, increased frequency of convulsion, respiratory depression, rash, and any other side effects were noted. When one drug either Phenobarbital or levetiracitum failed to control convulsion alone in a patient then that patient was treated as institutional protocol (Phenobarbital Fosphenytoin Midazolam and so on). Drugs were purchased from local market and all the levetiracetam were from same manufacturer and which is also same in case of Phenobarbital. For statistical analysis Independent sample t-test and chi-square test were used and $p$ value $<0.05$ was considered significant. Statistical software used for analysis of data was SPSS version 20.

\section{Results:}

In this study 69 patients reached randomization and out of them 34 were allocated to intervention group and 35 were allocated for control group. Finally, 34 from intervention group and 35 from control group was analyzed (Fig.-1). Most of the patients were normal in birth weight category and it was $94 \%$ and $86 \%$ in intervention and control group respectively. Most of the patients were delivered by normal vaginal delivery in both groups. It was $68 \%$ and $77 \%$ in intervention and control group respectively. Seizure developed in most of the patients within 12 hours of birth. In intervention group $74 \%$ and in control group $71 \%$ neonates developed seizure within 12 hours (Fig.-2). Most of the patients got admitted within 12 hours of age, among them $62 \%$ were in intervention group and $63 \%$ in control group. Among the four basic types of seizure clonic type was predominant in both groups. It was $56 \%$ and $49 \%$ in intervention and control group respectively followed by subtle type which was $32 \%$ in intervention group and $37 \%$ in control group. There was no myoclonic type seizure in any of the group (Fig.-3). 


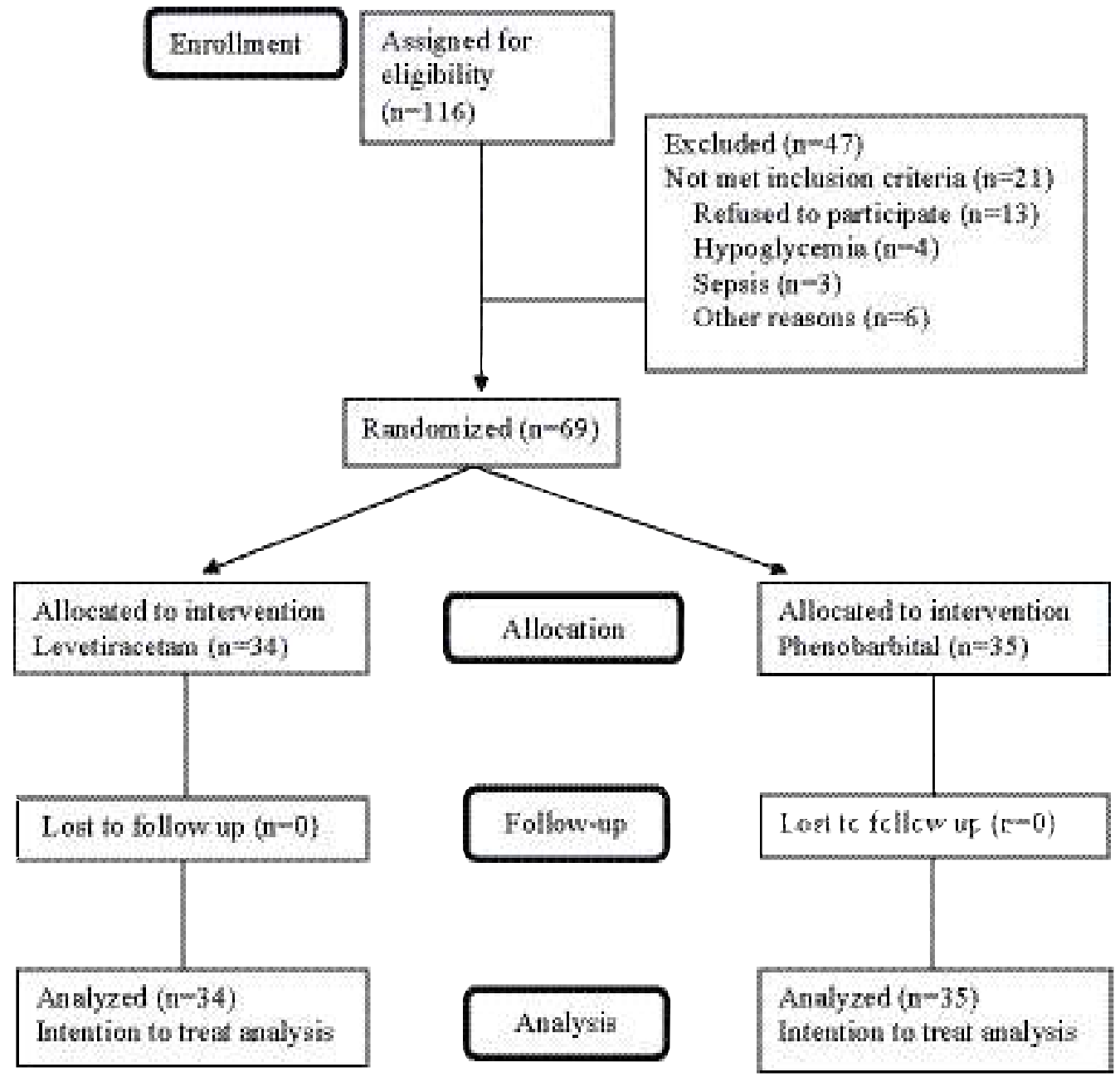

Fig.- 1: Diagram of flow of participants in each stage of the study.

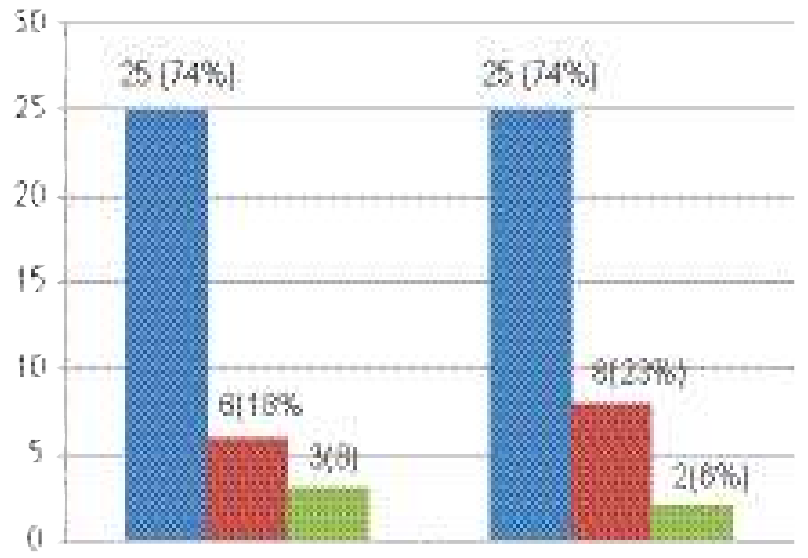

Iriteryenthin iscup

Consol iscup
[L $92 \mathrm{hir}$
$812.4 \mathrm{hr}$
籍> 2 h

Fig.-2: Age at seizure onset

Fig.-3: Seizure type in patients 
Table-I

Baseline characteristics of study population

\begin{tabular}{lccc}
\hline Baseline variable & Intervention 3 group & Control group & p value \\
\hline Gestational age wk & $38.29 \pm 1.11$ & $38.31 \pm 0.99$ & 0.983 \\
Birth weight g & $2862.05 \pm 323.38$ & $2885.71 \pm 400.81$ & 0.067 \\
Male sex & $20(58.82)$ & $21(60)$ & 0.921 \\
Normal vaginal delivery & $23(67.64)$ & $27(77.14)$ & 0.377 \\
Antenatal checkup done & $21(61.76)$ & $23(65.71)$ & 0.733 \\
Outborn patients & $20(58.82)$ & $22(62.85)$ & 0.731 \\
Age at admission hr & $10.13 \pm 10.08$ & $9.90 \pm 9.53$ & 0.724 \\
Age at seizure onset hr & $9.39 \pm 8.56$ & $9.82 \pm 8.72$ & 0.791 \\
\hline
\end{tabular}

Numerical variables are expressed as mean $\pm \mathrm{SD}$, and categorical variables as numbers (\%)

Table -II

Treatment outcome of study population $(n=69)$

\begin{tabular}{lccc}
\hline & Intervention group & Control group & p value \\
\hline Seizure control & $24(70.58)$ & $14(40)$ & 0.011 \\
Need for more than 1 drug & $10(29)$ & $21(60)$ & 0.011 \\
Adverse effect & $3(8.82)$ & $15(42.85)$ & 0.001 \\
Length of hospital stay, Day & $6.00 \pm 1.98$ & $8.03 \pm 2.82$ & 0.340 \\
\hline
\end{tabular}

Seizure control was significantly higher $(p=0.011)$ in intervention group $(71 \%)$, but it was $40 \%$ in control group and need for more than one drug was significantly lower in intervention group $(p=0.011)$. Adverse effects were significantly lower in intervention group $(p=0.001)$, which was $9 \%$ in intervention group and $43 \%$ in control group and no life threatening adverse effect was observed in both the groups. There was no significant difference in terms of hospital stay in both the groups but mean hospital stay was less in intervention group in comparison to control group (Table-II). After predefined period of follow up ( 48 hours), finally $68 \%$ patients of intervention group and $69 \%$ patients of control group were discharged with advice.

\section{Discussion:}

Neonatal seizure has wide variety of causes but in this study we focused on neonatal seizure due to perinatal asphyxia, the most common cause.

In baseline data, male sex was found predominant in comparison to female in both groups. It is probably due the social custom of our country where still male offspring is more preferred and taken care of by the parents and family members and they are less interested to spend time and money for female offspring by taking them to distant higher level hospitals. In this study it was found that with a comparable baseline characteristics levetiracetam has significantly higher efficacy in comparison to phenobarbital ( $71 \%$ vs $40 \%)$ in controlling neonatal seizure due to perinatal asphyxia. Furwentsches et al. on their pilot study on 6 neonates with seizure used levetiracetam $10 \mathrm{mg} / \mathrm{kg} /$ day loading increasing to $30 \mathrm{mg} / \mathrm{kg} /$ day over 3 days. All 6 patients became seizure free within 6 days. ${ }^{14}$ They used much lower loading dose than us, and used additional doses of phenobarbital during titration of levetiracetam dose and needed several doses and days to control seizure though they had $100 \%$ seizure control over 6 days, it is not clear whether this seizure control was attributed by phenobarbital or not. Whereas we used single loading dose of $50 \mathrm{mg} / \mathrm{kg}$ followed by $10 \mathrm{mg} / \mathrm{kg}$ maintenance 8 hourly and only immediate seizure control was taken into account. Khan et al in their retrospective study with levetiracetam as first or second line anticonvulsant reported $86 \%$ seizure control within one hour but some of them recurred 
so the actual control would be lower and for that we think that current study finding is comparable. ${ }^{5} \mathrm{Khan}$ et al. again in their retrospective study reported $82 \%$ seizure control in preterm neonates over 24 hours. It would be lesser if they only take into account those cases with immediate seizure control like this current study and would be comparable. ${ }^{15}$ Kirmani et al retrospectively reviewed neonates treated with 10$50 \mathrm{mg} / \mathrm{kg}$ loading of levetiracetam for seizure found $86 \%$ seizure control within one hour and $100 \%$ within 72 hours and we think our finding would be comparable if we take seizure control over one hour into account. ${ }^{16}$ Pharmacokinetic studies have established a benign safety profile for levetiracetam. Li et al. used initial loading doses of 15 to $40 \mathrm{mg} / \mathrm{kg}$ and demonstrated that levetiracetam was welltolerated in 18 neonates with seizures. The only adverse event present was somnolence. They also found linear kinetics, minimal protein binding and no hepatic metabolism with levetiracetam use in children and neonates. ${ }^{17}$ In our study a dose of $50 \mathrm{mg} / \mathrm{kg}$ was used as an initial loading dose and were maintained on $10 \mathrm{mg} / \mathrm{kg}$ every 8 hours and patients tolerated well. There are no clear dosing recommendations available currently in the literatures where doses range from 10 to $100 \mathrm{mg} / \mathrm{kg}$ were used. ${ }^{18-22}$ In terms of adverse effects levetiracetam was found much safer in comparison to phenobarbital. Adverse effects, were found in $9 \%$ cases of levetiracetam group and $43 \%$ in phenobarbital group. Most of the study with levetiracetam found no significant adverse effect and like us they also found somnolence as comparatively commoner insignificant side effect. 5,8,13,23, 24,

\section{Conclusion:}

Levetiracetam is more effective and safe in comparison to phenobarbital in the treatment of neonatal seizure due to perinatal asphyxia. Multicenter study with larger sample size, use of EEG, monitoring drug concentration and renal functions are recommended.

\section{References}

1. Cloherty JP, Eichenwald EC, Hansen AR, Stark AR. Manual of neonatal care, $4^{\text {th }}$ eds, Wolter Kluwer, Philadelphia 2012.

2. Velaphi S, Mokhachane M, Mphahlele R, BackhA. Effect of prophylactic Phenobarbital on seizures, encephalopathy and mortality in neonates with Perinatal asphyxia. South African Journal of Child Health 2013; 7(1): 1-14.

3. Camfield CS, Chaplin S, Doyle AB, Shapiro SH, Cummings C, Camfield PR. Side effects of Phenobarbital in toddlers; behavioral and cognitive aspects. Journal of Pediatrics 1979; 95(3): 361-5.

4. Ramantani G, Ikonomidou C, Walter B, Rating $D$, Dinger. Levetiracetam: Safety and efficacy in neonatal seizures. European Journal of Paediatric Neurology 2011; 15(1): 1-7.

5. Khan O, Chang E, Ciprini C, Wright C, Crisp E, Kirmani B. Use of intravenous levetiracetam for management of acute seizures in neonates. Pediatric Neurology 2011; 44(4):265-9.

6. Boylan GB, Rennie MD, Chorley G, Pressler $\mathrm{RM}$, Fox GF, Farrer $\mathrm{K}$ et al. Second-line anticonvulsant treatment of neonatal seizures: a video-EEG monitoring study. Neurology 2004; 62(3):486-88.

7. Gal P, Toback J, Boer HR, Erkan NV, Wells TJ. Efficacy of Phenobarbital monotherapy in treatment of neonatal seizure - relationship to blood level. Neurology 1982;32:1401-04.

8. Glauser TA, Mitchell WG, Weinstock A, Bebin M, Chen D, Cupez R, et al. Pharmacokinetics of Levetiracetam in infants and young children with Epilepsy. Epilepsia 2007; 48( 6): 1117-22.

9. Maitre NL, Smolinsky C, Slaughter JC, Stark AR. Adverse neurodevelopmental outcomes after exposure to phenobarbital and levetiracetam for the treatment of neonatal seizures. Journal of Perinatology 2013; 33(11): 841-86.

10. Manthey D, Asimiadou S, Stefovska V, Kindle AM, Fassbender J, Ikonomiadou C, et al. Sulthiame but not levetiracetam exerts neurotoxic effect in the developing rat brain. Experimental Neurology 2005; 193(2); 497-03.

11. Mikati MA, El Banna D, Sinno D, Mroueh S. Response of infantile spasms to levetiracetam, Neurology 2008; 12: 10-3.

12. Striano P, Coppola A, Pezzella M, Ciampa C, Specchio N, Rangona MM, et al. An open-label trial of levetiracetam in severe myoclonic epilepsy of infancy. Neurology 2007; 69(3): 250-4. 
13. Koppelstetter A, Bhrer C, Kaindl AM. Treating neonates with levetiracetam: A survey among German University Hospitals. Klinische Padiatri.2011; 223(7):450-2.

14. Fürwentsches $A$, Bussmanna $C$, Ramantania G, Ebinger F, Philippi $\mathrm{H}$, Poschl J, et al. Levetiracetam in the treatment of neonatal seizures: A pilot study. Seizure 2010;19(3): 185-9.

15. Khan O, Chang E, Ciprini C, Wright C, Crisp E, Kirmani B. Role of intravenous levetiracetam for acute seizure management in preterm neonates. Pediatric Neurology 2013; 49(5): 340-3.

16. Kirmani BF, Crisp ED, Kayani S, Rajab H. Role of Intravenous Levetiracetam in Acute Seizure Management of Children.Pediatric Neurology 2009; 41(1): 37-9.

17. Li J, Xiao N, Chen S. Efficacy and tolerability of levetiracetam in children with epilepsy. Brain Development 2011; 33(2): 145-51.

18. Mikati MA, El Banna D, Sinno D, Mroueh S. Response of infantile spasms to levetiracetam. Neurology 2008; 12: 10-3.

19. Gorayra JS, Khurana DS, Valencia I, Melvin JJ, Cruz M, Legido A, Kothare SV. Intravenous levetiracetam in children with epilepsy. Pediatric Neurology 2008; 38(3): 160-70.

20. Silverstein FS, Ferriero DM. Off-label use of antiepileptic drugs for the treatment of neonatal seizures. Pediatr Neurology 2008; 39(2): 77-9.

21. Cilio MR, Bianchi R, Balestri M, Onofri A, Giovannini S, Capua M, et al. Intravenous levetiracetam terminates refractory status epilepticus in two patients with migrating partial seizures in infancy. Epilepsy Research 2007. 86(1).66-71.

22. $\mathrm{Ng} \mathrm{Y,} \mathrm{Hastriter} \mathrm{EV,} \mathrm{Javier} \mathrm{F,} \mathrm{Khoury} \mathrm{EM,}$ Chapman KE. Intravenous levetiracetam in children with seizures: a prospective safety study. Journal of Child Neurology 2010; 25(5): 551-55

23. Opp J, Tuxhorn I, May T, Kluger G, WiemerKreul A, Kurlmann $G$, et al. Levetiracetam in children with refractory epilepsy: A multicenter open label study in Germany. Seizure 2005; 14(7): 476-84.

24. Abend NS, Gutierrez-Colina A, Monk HM, Dlugos DJ, Clancy RR.Levetiracetam for treatment of neonatal seizures. Journal of Child Neurology 2011; 26(4): 465-70 